CIC. Cuadernos de Información y Comunicación

ISSN: 1135-7991

http://dx.doi.org/10.5209/ciyc.64635

\title{
Medios públicos en Honduras: una propuesta de desarrollo y buenas prácticas $^{1}$
}

\author{
Amelia Argentina Espinoza Hulse ${ }^{2}$
}

Recibido: 1 de febrero de 2019 / Aceptado: 25 de mayo de 2019

Resumen. Esta investigación describe el nacimiento de los medios de propiedad pública en Honduras y su caracterización como servicios gubernamentales. El texto explica qué son los medios públicos y el servicio público y cuáles deberían de ser sus funciones dentro de la sociedad. Finalmente, se realiza una propuesta de cómo debe ser idealmente el modelo audiovisual público hondureño.

Palabras clave: Medios públicos, servicio público, medios gubernamentales.

\section{[en] Public media in Honduras: a proposal for development and good practices}

\begin{abstract}
This paper describes the birth of public owned media in Honduras and its characterization as government services. The text explains what is public service media and public interest and what should their functions be within society. Finally, a proposal of a new Honduran model of public service media is presented.
\end{abstract}

Keywords: Public service media, Public service, Governmental media service.

Sumario: 1. Introducción. 2. Definiciones. 3. Metodología. 4. Órganos reguladores. 5. Medios de propiedad pública. 5. Nuevo modelo de medios públicos en Honduras. 6. Conclusiones. 7. Referencias bibliográficas.

Cómo citar: Espinoza Hulse, A.A. (2019). Medios públicos en Honduras: una propuesta de desarrollo y buenas prácticas, en CIC. Cuadernos de Información y Comunicación 24, 95-112.

\section{Introducción}

Construir día a día una nación y mantener cada una de sus partes juntas y en funcionamiento como un engranaje es responsabilidad principal del Gobierno con la participación y vigilancia de la ciudadanía que asegura que se cumplan y se cubran las necesidades prioritarias de la gran mayoría. Para efectuar esta misión integradora se debe de hacer uso de múltiples herramientas y recursos, uno de ellos son los medios de servicio público (MSP) (entiéndase radio, televisión, prensa y sus extensiones a través de la red) que fungen como plataformas catalizadoras para proveer un servicio

\footnotetext{
$1 \quad$ Elaborado en el marco del módulo de Jean Monnet, "The role of public service media in promoting active citizenship and inclusive dialogue" (586770-EPP-1-2017-1-ES-EPPJMO-MODULE).

2 Universidad Complutense de Madrid.
} 
que ayude a cubrir las necesidades morales, comunicacionales, sociales y culturales prioritarias del país, tomando en cuenta la pluralidad y los diversos intereses de las comunidades y etnias que convergen en la nación.

Es así como surge el interés por revisar la situación actual de las plataformas audiovisuales públicas hondureñas por medio de un análisis crítico de dichas instituciones con el fin de proponer cambios en su modelo audiovisual para que sea de beneficio para toda la población. Para ello es necesario conocer cómo surgen estos medios gubernamentales y con qué fines, identificar los entes que tienen una injerencia, control y participación sobre dichos medios y finalmente definir buenas practicas que llevarán a los medios gubernamentales a convertirse en medios de servicio público.

\section{Definiciones}

\subsection{Medios públicos frente a medios gubernamentales o estatales}

Es necesario definir algunos conceptos teóricos básicos para comprender qué son los medios públicos (public service media) y cómo se diferencian de los medios gubernamentales o estatales, esto servirá de base para comprender qué tipo de medios se tienen en Honduras y si se está cumpliendo la labor de fomentar la participación ciudadana, democratización de la comunicación, diversidad, pluralismo y garantizar el derecho y acceso a la comunicación e información.

Según lo expuesto por el experto latinoamericano Guillermo Mastrini (2000), de manera generalista los medios públicos tienen principalmente dos modelos de estructura: el primero es el modelo estadounidense donde el gobierno otorga licencias a los medios para que estos los administren y el segundo es el modelo europeo donde bajo el amparo de normativas y regulaciones los medios son gestionados públicamente y sin fines de lucro. En América Latina la televisión pública combina elementos de la televisión estatal, gubernamental y comercial, el Gobierno tiene influencia y control del ente y sus contenidos son elaborados con criterios comerciales. A la vez, en su publicación "Medios públicos y derecho a la comunicación”, plantea (2011):

Los medios de comunicación y en particular los servicios de comunicación audiovisual no aparecen, ni conceptual ni prácticamente, involucrados al derecho ciudadano de contar con medios públicos que representen al conjunto de la sociedad. Por otra parte, sí existen numerosos medios de propiedad estatal, que en la mayoría de los casos han quedado limitados a tornarse voceros de los intereses del gobierno. De esta forma no se ha sabido/podido construir un vínculo con la ciudadanía que permita que los medios públicos sean concebidos como una alternativa válida para la difusión de información de calidad, de programas que respondan a la diversidad cultural de nuestros países, y generen ficciones que se vinculen con las identidades presentes en nuestras regiones.

Los medios de servicio público tienen el reto de ofrecer a toda una diversa población una nueva propuesta en educación, información y entretenimiento. Tienen el compromiso puesto que los medios públicos son de todos y deben por ende contrarrestar los paradigmas y esquemas culturales que imponen los medios comerciales neolibera- 
les, fomentando y construyendo una cultura de vida y paz en la nación. Lamuedra (2012:17) compila que una radiotelevisión pública líder, que funja como un referente y que tenga como característica principal la aprobación y confianza de la población puede influir positivamente en todo el sistema mediático y que el primer argumento sobre la necesidad de contar con una radiotelevisión publica se centra primordialmente en que estas plataformas son bases para mantener las sociedades democráticas.

Sin embargo, el principal cuestionamiento y pérdida de credibilidad de los medios públicos según Mastrini (2000) es que estos medios de comunicación han sufrido una ininterrumpida dependencia de los gobiernos en turno, sean estos de dictadores o democráticos, y como consecuencia la población ha identificado a estos medios como las plataformas para difundir la propaganda de los políticos. A su vez, el Estado invierte muy poco para desarrollar estos medios y fortalecer la esfera pública, tal es el caso de los medios nacionales hondureños donde no se realizan concursos públicos para nombrar a sus directivos con el fin de asegurar que posean la formación y conocimientos para liderar los medios públicos, tampoco se tienen todos los recursos y herramientas para ejecutar el trabajo diario y entre otros puntos, no se realizan investigaciones de mercado para conocer los gustos y necesidades de la ciudadanía. Es importante destacar que los medios comerciales y privados se posicionaron primero en la nación y décadas después, se fundaron los medios gubernamentales, por otra parte, los medios comunitarios han tenido poca participación por la falta de financiación y legislación.

El italiano-venezolano Antonio Pasquali (1991) propone que los medios públicos deberían de basarse en una doble independencia: económica y política. Económica en el sentido de que en muchos casos los medios de comunicación obtienen sus fondos por medio de la venta publicitaria o patrocinio de espacios y que lo ideal es que estos medios no dependan de la recaudación publicitaria, por lo que propone que para que exista una identidad entre la sociedad y los medios de servicio público los ciudadanos sean quienes paguen y subvencionen dichos medios. Aplicándolo al contexto hondureño, dado el alto índice de pobreza y crisis políticas a las que la nación ha estado expuesta, una propuesta como esta sería inviable en estos momentos y en lugar de crear esa relación medio público-ciudadanía se generaría una ruptura y descontento. La independencia política significa que los medios públicos no pueden ser controlados por el gobierno de turno, para que este no sea cuestionado como un medio gubernamental oficialista. A su vez, Antonio Pasquali (1991b:172) desde el siglo pasado define en síntesis cómo debe ser idealmente un medio público:

Una radiodifusión puede definirse pública cuando cumple con este pequeño decálogo: es independiente, participativa y de alta calidad; es de alcance nacional; respeta la diversidad de gustos del público; practica todos los géneros sin limitarse a lo populista o a lo elitesco; regionaliza sus emisiones y fomenta la circulación de mensajes periferia centro; está técnicamente a la vanguardia; produce al menos un $40 \%$ de la programación que difunde; es complementaria y no competitiva; asegura coproducción e intercambios regionales e internacionales. Pero por encima de todo, una radiodifusión es pública si se alimenta mayoritariamente de fondos públicos.

Tomando todos los planteamientos como base se puede definir hoy en día que un medio de servicio público es aquel que conoce las necesidades de la ciudadanía ya que periódicamente realiza investigaciones de mercado y que desde su plataforma busca 
brindar una solución o ruta para resolver las mismas, para realizar esta acción es necesario que el medio tenga una independencia editorial, política y económica ya que si no lo posee no podrá trabajar de manera autónoma y estará respondiendo a los intereses de los grupos de poder. Por otra parte, deberá de contar con códigos deontológicos y la participación activa de ciudadanía para proteger y asegurar el bien común.

Es así donde se plantea el cuestionamiento retórico, ¿cumplen los medios hondureños con estos criterios? ¿La radio y la televisión pública es independiente del gobierno? ¿Se realizan estudios de mercado para conocer los gustos de la ciudadanía? ¿En las parrillas de programación de los medios públicos hondureños se ofrece contenido variado de diversos géneros? ¿Tienen estos medios públicos cobertura nacional y regionalizan sus emisiones? ¿Son los medios públicos hondureños líderes en materia de comunicaciones? ¿Sus producciones son prioritariamente nacionales con el fin de fortalecer la identidad y cultura catracha? Y finalmente ¿Se realizan coproducciones e intercambios con el fin de crear alianzas estratégicas?

\subsection{Servicio público e interés público}

Existen diversas definiciones de servicio público y según Antoni (2006) este inicia en el Estado social de derecho en la antigua ciudad griega:

Los primigenios conceptos de libertad, justicia y equidad alumbran una organización social política que es irrealizable sin la articulación de una serie de servicios públicos esenciales, dichos servicios eran prestados por un conjunto de funcionarios elegidos por la Asamblea donde los tribunales atenienses eran la piedra angular de la democracia griega.

Según varios académicos el servicio público tuvo su auge o se popularizó en Francia donde la ciudadanía recibió derechos políticos y el Estado debía de asegurar dichos compromisos. Los principios de libertad, igualdad y fraternidad engloban que las políticas públicas de interés general deberían de ofrecerse a toda la ciudadanía en igualdad de condiciones y de forma ininterrumpida. Vidal (2006:26) afirma:

En 1873 el Tribunal de Conflictos francés emite el llamado Fallo Blanco en virtud del cual la noción de servicio público sucede a la del poder público como fundamento del derecho administrativo francés. Según esta sentencia la administración se ha de regir por normas diferentes de las que se aplican en las relaciones de los particulares... El concepto de servicio público se convertirá en el principal criterio de aplicación del derecho administrativo.

¿Cómo definir entonces qué es el servicio público? Para Gaëtan Tremblay (1988) el servicio público podría definirse como referencia de cuatros servicios:

1. Tipo de actividades: Relacionadas con el interés general de una colectividad, y la interpretación de lo que es "interés general" varía según sociedades, ideologías y épocas.

2. Criterio orgánico: Remite al agente que posee la responsabilidad y control de la gestión de actividades de interés general (intervención necesaria del Estado pero también puede entregarse a una empresa privada). 
3. El criterio formal: Intención del legislador que decide sobre el servicio público y se puede fundamentar en la importancia de la actividad respecto al bien común, las lagunas del sector privado ante la obtención de algunos objetivos colectivos y la forma y grado de control que se considera necesario

4. El régimen jurídico: El servicio público puede estar o no sujeto a un régimen de derecho común o de derecho especial.

En síntesis, Tremblay (1988:57) define que:

El servicio público es una actividad considerada de interés general por una colectividad y como tal reconocida por el Estado. Dicha actividad no puede abandonarse absolutamente a la actividad privada y las leyes del mercado y por lo tanto el Estado asume su responsabilidad y su control sometiéndolo si fuera necesario a un régimen jurídico especial.

Por otra parte, el interés público está relacionado con las funciones políticas, sociales y económicas de toda la sociedad, las cuales deben de estar sustentadas por instituciones políticas-democráticas y poseer una conexión con la libertad de prensa y publicación. (McQuail, 2003:185)

Asimismo, organismos internacionales ponen un foco de atención en los medios de servicio público, "UNESCO defines PSM as those media that fulfill the same public purposes as public service broadcasters, but use digital media and platforms, including the Internet, instead of broadcast television or radio" (Mediadem, 2012). También la UNESCO establece en su manual de buenas practicas (2006:5) que la televisión y la radio siguen siendo las plataformas de comunicación e información más accesibles para grupos minoritarios y es por ello que se requiere que estos medios "sirvan a todas las poblaciones, reflejando las necesidades, preocupaciones y expectativas de las diversas audiencias, sin considerar su tamaño. La radiotelevisión de servicio público es un elemento esencial para asegurar la pluralidad, la inclusión social y el reforzamiento de la sociedad civil". Si estas plataformas son bien administradas tienen la capacidad de proteger los intereses de los ciudadanos ya que su rol principal es el de proporcionar acceso y participación en la vida pública facilitando el acceso a la educación y cultura.

El Consejo Europeo, citado por Mediadem (2007:14), "considera que la primera prioridad para los MSP debe ser garantizar que su cultura, políticas, procesos y programación reflejen y garanticen la independencia editorial y la autonomía operativa". Para ello la misión de servicio público de estos canales debe de definirse con el objetivo que sus contenidos televisivos aborden temas con esta orientación y sean difundidas en todas sus plataformas. El grupo de especialistas en servicio público (2007:31) cita a Carpentier al establecer cuatro obligaciones para los medios de servicio público:

1. Obligación informativa y de control: La información siempre es clasificada y seleccionada, nunca es neutral, por ello los MSP deben de tener un ojo crítico.

2. Obligación de representación: Los MSP deben de evitar interpretaciones erróneas y estereotipos asegurando así la justicia para que exista una cohesión en la sociedad.

3. Obligación de foro: Se refiere a los servicios y actividades que se realizan para fortalecer la cultura democrática. 
4. Obligación participativa: elemento nuclear para ampliar la participación individual en la democracia, que la ciudadanía pueda influenciar en la creación de contenidos. Participación en los medios y participación a través de los medios públicos.

Ana María López (2015:23) plantea que describir un modelo de medio público no es tarea sencilla y que en términos teóricos se podría describir un modelo ideal o utópico. Define un servicio público como, "servicio prestado regularmente a la sociedad para la satisfacción de una necesidad y desde esa perspectiva, la información se convierte en una necesidad y los medios de comunicación públicos en un servicio para hacerla efectiva".

Pero ¿Cómo definir exactamente qué es un medio de servicio público? (Nowak y Kuhn, 2015:3) lo detallan en forma de cuestionamiento: ¿Cuáles son los rasgos y cualidades diferenciadoras entre un medio comercial y uno público?, ¿pueden los MSP definirse como medios alternativos que ofrecen un contenido diverso en sus plataformas con cierta cantidad de valor público indistintamente si son públicos, comerciales o comunitarios en términos de propiedad?, o ¿son los MSP simplemente repetidoras que son definidos como públicos porque cuentan con un decreto o están bajo el amparo de una legislación especial? No hay respuestas únicas para estos cuestionamientos ya que existen medios de comunicación privados o comerciales que realizan trabajos de servicio público y en contraste hay medios públicos que solamente proveen a la ciudadanía de entretenimiento y sus contenidos no se distinguen de los medios comerciales. En tal sentido, una definición de medio de servicio público queda supeditado a quien lo define bajo su contexto legal, social, cultural, político y nacional, sin embargo, la premisa predominante debe ser el de servicio público, como una plataforma de difusión que se dirige a todos los ciudadanos bajo igualdad.

\section{Metodología}

El presente artículo sobre los medios públicos en Honduras se construyó en base a una metodología cualitativa ya que se recolectó y analizaron fuentes de información documental (noticias, normativas, leyes, libros de expertos, entre otros) y para complementar y corroborar dicha información existente, se utilizó el método inductivo recurriendo a entrevistas semiestructuradas aplicando un cuestionario con preguntas abiertas que fueron respondidas por tres actores importantes: el jefe del espectro radiofónico de CONATEL (Ing. Fausto Zambrano), el productor general de Televisión Nacional de Honduras (Lic. Marco Centeno) y el director de Radio Nacional de Honduras (Lic. Cristiam Martínez), generando así información contextual que se ha plasmado a los largo de todo este artículo. El cuestionario se aplicó en junio del 2018 y la duración promedio de cada una fue de 15 minutos. Dentro de los contenidos planteados y expuestos por los actores de los medios públicos, se les cuestiono sobre el nacimiento del medio de comunicación, su propósito de creación, forma de financiación del medio, modelo de gestión administrativa, códigos y manuales existentes, líneas estratégicas, entre otras. En el caso del responsable de Conatel se conoció cuáles son las funciones del ente y sus políticas de operación. 
A continuación, se detalla el análisis de la información recolectada y se describe cada uno de los actores destacados que influyen en las plataformas de servicio público audiovisual hondureño.

\section{4. Órganos reguladores}

\subsection{Comisión Nacional de Telecomunicaciones (CONATEL)}

La Comisión Nacional de Telecomunicaciones (CONATEL) fue fundada el 5 de diciembre de 1995, mediante decreto 185/95. Es un organismo estatal desconcentrado que ejecuta, mediante la regulación y coordinación, la política de telecomunicaciones en la República de Honduras. Colabora con el Presidente de la República de Honduras en la formulación de las políticas de Telecomunicaciones. (Telecomunicaciones, 2017)

El Jefe del Espectro Radiofónico el Ing. Fausto manifiesta que CONATEL tiene como misión impulsar el acceso universal para fomentar y potenciar la industria y reducir la brecha digital por medio de la competencia, inclusión social y promover la calidad de los servicios. Existen diversas regulaciones específicas para la operación y políticas de CONATEL, algunas de estas son la Ley Marco del Sector de Telecomunicaciones, Ley Especial Sobre Intervención de Comunicaciones Privadas, Ley de Portabilidad Numérica, Reglamento General de la Ley Marco del Sector de Telecomunicaciones, entre otros.

El Sistema Integrado de Telecomunicaciones y Administración del Espectro (SITAE) de la Comisión Nacional de Telecomunicaciones tiene como objetivo primordial automatizar los procesos fundamentales que constituyen los pilares para que la institución cumpla con su misión de: "promover el desarrollo, modernizar, democratizar y humanizar el sector de las telecomunicaciones en Honduras, fomentando la participación de la inversión pública y privada en el sector, dentro de un ambiente libre y de leal competencia, velando por la protección de derecho de los usuarios y la universalidad del acceso al servicio" (Espectro, 2017).

Se trata de que el sistema propicie las interrelaciones de las diferentes áreas de CONATEL, en un marco de cooperación, seguridad y confiabilidad, para lograr los diversos cometidos de la Institución; agregándose como actores externos y participativo, tanto los usuarios y los operadores de los diferentes servicios de telecomunicaciones existentes en el país, como las demás instituciones gubernamentales y los organismos internacionales relacionados con el sector de las telecomunicaciones.

El SITAE permite a CONATEL realizar las siguientes funciones:

- Investigar e identificar los transmisores de radio que operan sin licencia (ilegales).

- Investigar e identificar las fuentes de interferencia que afectan la correcta operación de los equipos que pertenecen a los titulares de las licencias de frecuencias radioeléctricas.

- Comprobar que los transmisores de radio aprobados operan conforme con la licencia recibida.

- Obtener información sobre la ocupación del espectro radioeléctrico.

- Realizar la administración del espectro radioeléctrico: lo que incluye los estudios de asignación de frecuencias a nivel de ingeniería (de compa- 
tibilidad electromagnética), así como el control de las tarifas y multas por pago de canon radioeléctrico, el control de las solicitudes de uso del espectro radioeléctrico, la emisión de las licencias de uso del espectro correspondiente y emisión de certificados de homologación de equipos. (SITAE,2017)

La injerencia que este ente tiene sobre los medios públicos es únicamente en el área técnica, asignado las frecuencias correspondientes en televisión (TNH) y radio (RNH) con sus respectivos permisos de operación.

\subsection{Despacho de Comunicación y Estrategia Presidencial}

Creado bajo el nombre de Despacho de Comunicación y Estrategia Presidencial en el primer mandato del Presidente Juan Orlando Hernández en el 2014, fue liderado por su hermana la Ingeniera Hilda Hernández (QDDG) a quien se nombró como Ministra Directora del Despacho de Comunicación y Estrategia. Dicho nombramiento fue cuestionado dado que la Constitución de la República de Honduras establece en su artículo 250 (1982) "No pueden ser secretarios del Estado: 1. Los parientes del presidente de la República, dentro del cuarto grado de consanguinidad y segundo de afinidad". ${ }^{3}$

Sin embargo, este despacho no es una Secretaría de Estado, sino que funge como una unidad de asesoría y su Ministro Director es un estratega en materia de comunicación. La diferencia entre Ministro y Secretario según explicaciones del Presidente de la República de Honduras radica en que un Ministro no tiene asignado un presupuesto o cartera y un Secretario sí. Este despacho opera con el presupuesto general de la Secretaría de Estado de la Presidencia del Gobierno de la República de Honduras y coordina todos los medios y plataformas gubernamentales, al ser una unidad de asesoría directa de Casa Presidencial no posee un decreto legislativo de creación.

Al presente la Ministra Directora del Despacho de Comunicación y Estrategia Presidencial es María Andrea Matamoros Castillo, hija del actual presidente del Tribunal Supremo Electoral (TSE), David Matamoros Batson. Dicho despacho tiene como misión y propósito, "el diseñar las estrategias de comunicación estatales para garantizar a la población el acceso a la información de todo el accionar del Gobierno y contribuir a una administración pública transparente" (Despacho de comunicación y estrategia, 2018). Este despacho funge a su vez como el canal para fortalecer las políticas públicas e iniciativas implementadas por el Gobierno.

El Despacho de Comunicación y Estrategia Presidencial orienta y dirige las líneas estratégicas de comunicación oficial del Gobierno utilizando como plataformas de difusión los medios de comunicación, entre ellos Radio Nacional de Honduras y Televisión Nacional de Honduras. Esta unidad asesora de comunicación y estrategia ha existido desde los Gobiernos de junta militar en los años 70 y desde entonces ha recibido diversos nombres, algunos de ellos: Despacho de Prensa, Estrategia y Comunicaciones, Despacho Asesor de Prensa, Despacho de Comunicaciones, entre otros. Dependiendo del Gobierno en turno se le asigna su nombre y funciones espe-

Modificado por Decreto 207/1987 y ratificado por Decreto 95/1988. Modificado por Decreto 248/1989 y ratificado por Decreto 4/1990". 
cíficas. La relación de regulación de estos órganos sobre los medios públicos se resume en la figura 1.

Figura 1. Órganos reguladores e interrelación medios públicos.

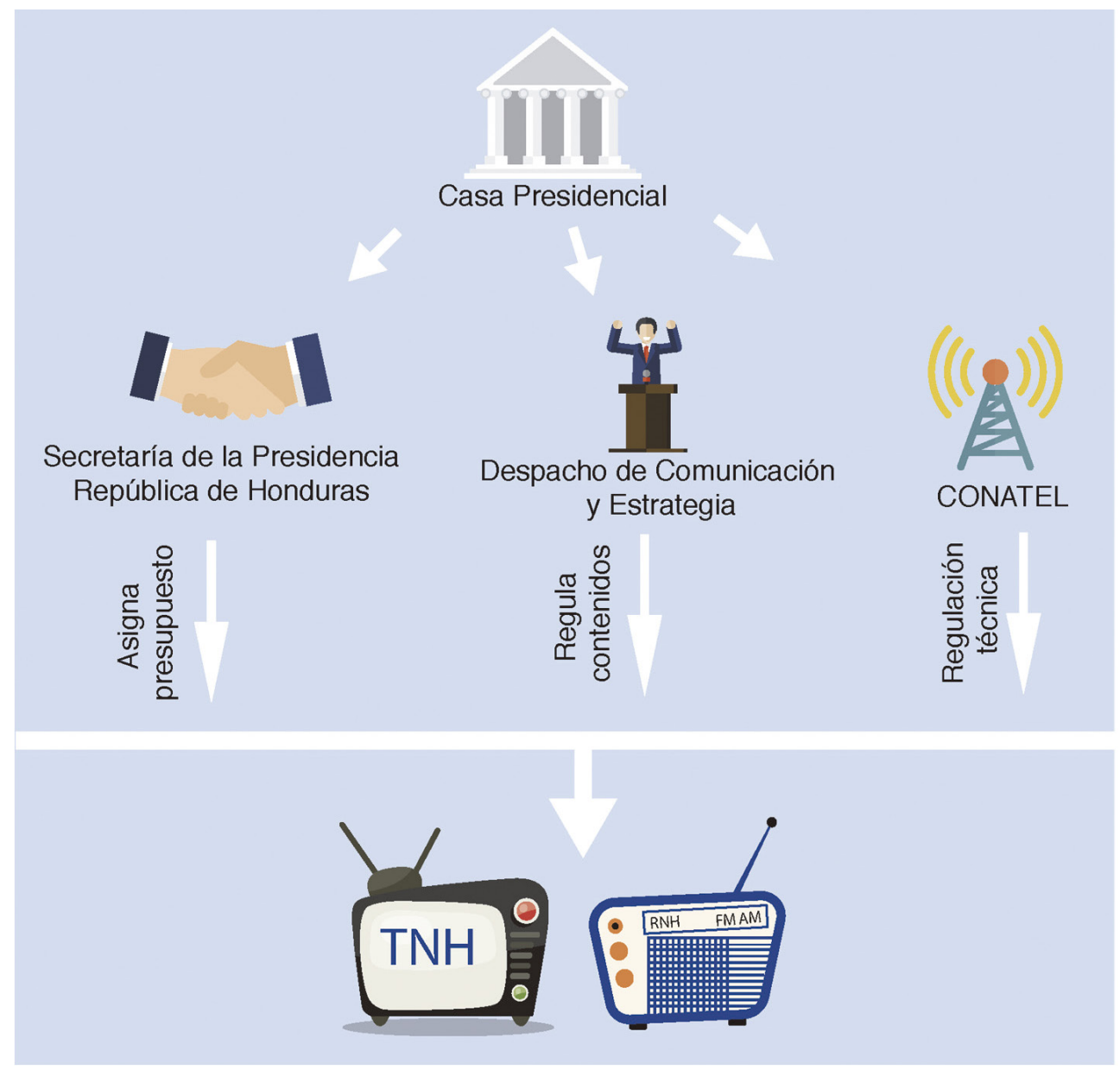

Fuente: Elaboración propia (vectores extraídos de freepik)

\section{Medios de propiedad pública}

\subsection{Radio Nacional de Honduras (RNH)}

El catedrático hondureño Mauricio Pineda relata en su artículo "El futuro de la Televisión" (2009) que la radio llega a Honduras en el año de 1921, su propietaria era la empresa bananera Tela Railroad Company de capital mayoritariamente estadounidense. Luego de esto nacen muchos otros canales y radios privadas y comerciales en el país. 
El Director de Radio Nacional de Honduras, el Lic. Cristiam Martínez narra que la radio nace en los Gobiernos de junta militar a mediados de los 70, pero habría que esperar hasta el 12 de marzo de 1976 para salir al aire como una iniciativa de los Gobiernos de junta militar siendo en ese momento Jefe de Gobierno el extinto General Juan Alberto Melgar Castro y al frente de la Secretaría de Prensa estaba el periodista Germán Allan Padgett, quien fue el principal impulsor de este medio de comunicación gubernamental. En un principio los estudios de Radio Honduras se ubicaron en el Instituto Nacional Agrario (INA) y luego se trasladaron al Estado Mayor Conjunto de las Fuerza Armadas de Honduras, posteriormente crean la Secretaría de Cultura, Turismo e Información (misma que actualmente ya no existe) y se trasladó la radio a esa dependencia. Finalmente, Radio Honduras se traslada a la Secretaría de la Presidencia de la República y actualmente depende directamente de la Presidencia de la República a través del Despacho de Comunicación y Estrategia.

Durante la década de los años 70 hasta principios del 2000, la Radio poseía una cobertura nacional y era reconocido por la producción de contenidos de calidad entre ellos dramas de obras de literatura hondureña, radio novelas, comedias, contenidos educativos, noticias gubernamentales y, hasta mediados de la década de los ochentas era el medio oficial de los partidos de la Liga Nacional de Fútbol, por todo lo anterior llegó a posicionarse en los primeros lugares de audiencia.

Actualmente RNH no posee un decreto legislativo de creación y su cobertura se limita a las ciudades de Tegucigalpa, San Pedro Sula, Santa Bárbara, Copán, Ocotepeque y Lempira (Casa Presidencial, 2018). Al igual que TNH, Radio Nacional depende de una partida presupuestaria que le asignan los administradores de Casa Presidencial, no vende publicidad ya que la ley no se lo permite. El Director de RNH es electo directamente por el Presidente de la República o el Secretario de Prensa o la Ministra de Comunicación y Estrategia en turno, el organigrama de la radio es vertical.

El primer medio gubernamental ha venido en decadencia en los últimos años debido a los recortes presupuestarios y alianza con TNH donde comparten la mayor parte de los recursos y administración, a esto se agregado que no se realizan investigaciones de mercado para conocer los gustos, preferencias y necesidades de la población dados los altos costos, tampoco dichos medios públicos aparecen en el listado de publicaciones de mediciones de rating del país, en su informe anual se establece (RNH, 2018:21)

Es evidente que el mayor problema en Radio Honduras ha sido y es; la falta de inversión en renovación de equipos y en mantenimiento de sus repetidores, y no tanto en su programación, dado que su nivel de audiencia está directamente ligado al rango de cobertura y alcance nacional que tenga la radio, y ese alcance nacional solo se logra con repetidores en buen estado.

La falta de interés o bien la falta de conocimiento de la importancia que tienen los medios públicos en una nación para asegurar la libertad de expresión, promover la participación ciudadana, asegurar el acceso a la información y democratización de la sociedad es evidente dado el poco interés, apoyo financiero y logístico que se da a estas plataformas. 


\subsection{Televisión Nacional de Honduras (TNH)}

La televisión en Honduras aparece en 1959 con el primer canal, Compañía Televisora Hondureña S.A. por medio de una asociación entre los empresarios de grandes corporaciones y cadenas televisivas estadounidenses (ABC TV Network) y empresarios locales del país (Fernando Lardizábal, Miguel Brooks, Rene Sempé y Raúl Zelaya) (Op cit.). Tras la llegada de la televisión comercial a Honduras, 48 años después nace la televisión gubernamental. Inicia sus trámites de creación en el 2007 en el Gobierno del ex presidente Manuel Zelaya Rosales, mediante Decreto Legislativo No. 35-2008 (publicado en el Diario Oficial La Gaceta el lunes 23 de junio de 2008) y en agosto del 2008 inicia sus operaciones bajo el eslogan, "La red informativa del Poder Ciudadano de Honduras".

Durante este tiempo y por los próximos 6 años se mantiene un conflicto legal por la frecuencia del Canal 8 donde en el 2008, "La Corte de Apelaciones de lo Contencioso Administrativo desautoriza al Gobierno el uso de la frecuencia del Canal 8 y la otorga a la empresa Teleunsa, propiedad del empresario Elías Asfura" (La Prensa, 2008).

Finalmente, en el 2014 la Sala de lo Constitucional de la Corte Suprema de Justicia emite sentencia definitiva, declarando que no hay lugar de un recurso de inconstitucionalidad contra el Decreto Legislativo 161-2010 y que las frecuencias 8 y 20 pertenecen al Estado. El Abogado Roberto Zacapa entonces Secretario General de la Secretaría de la Presidencia lee el comunicado oficial del Gobierno que manifiesta (La Prensa, 2014):

... Se ratifica que el espectro radioeléctrico es un recurso natural del Estado y por lo tanto, no puede ser disputado por un particular, sea persona natural o jurídica, porque el Estado de Honduras es el dueño del espectro radioeléctrico y ejerce un dominio predominante sobre el mismo. El Estado emite a través de la Comisión Nacional de Telecomunicaciones (Conatel), con concesiones temporales para ser utilizados por los medios de comunicación y en ese sentido, el primer licenciatario del rango de frecuencia de canal 8, fue siempre el Estado de Honduras.

Durante el lanzamiento y funcionamiento de Canal 8, "La red informativa del poder ciudadano de Honduras", en el año 2008 bajo el mandato del ex presidente Manuel Zelaya se observaba continuamente la propaganda política de la "cuarta urna", una iniciativa que buscaba el continuismo de Zelaya en el poder por medio de una reforma a la Constitución de la República (La Prensa, 2009).

No es el primer caso donde la televisión "pública" nace con propósitos políticos, propagandísticos y no como un servicio público o bajo la premisa del interés público. Tal es el ejemplo de la televisión en España como afirma Semova (2012:78): "Hemos de tener en cuenta que la televisión en España existe durante varias décadas como parte del aparato propagandístico de un régimen autoritario y por eso la dependencia gubernamental será una de las características constantes".

Honduras aún no cuenta con una legislación, estatutos o normativas de emisión de contenidos para este sector audiovisual dado que actualmente se han otorgado 388 licencias a canales de televisión y 1,075 licencias en FM y AM según la Comisión Nacional de Telecomunicaciones (CONATEL, 2018). 
En la actualidad el productor general de Televisión Nacional de Honduras manifiesta que dicho medio depende directamente del Despacho de Comunicaciones y Estrategia Presidencial, al pertenecer a Casa Presidencial es esta Secretaría la encargada de administrar y proveer los fondos para la operación del canal, a su vez, es allí donde se eligen a los directivos y se definen las líneas editoriales y estratégicas.

Este canal de televisión funge como la voz e imagen oficial del Gobierno de Honduras ya que es una plataforma para transmitir y dar a conocer los programas que ejecuta el Gobierno en turno. El modelo de gestión/administración de TNH está encabezado por la Ministra Asesora en el Despacho de Estrategia y Comunicación, quien elige y delega sobre el Gerente General de TNH. No poseen una junta directiva, tampoco se realiza un concurso público para contratar a su personal, sino que el Gobierno controla en su totalidad dicho medio público.

En TNH se rigen bajo el código de ética de Casa Presidencial y código de trabajo de Honduras. Mensualmente el personal ejecutivo elabora un informe de actividades que es presentado a la Gerencia General de TNH, mismos que anualmente entregan un informe al Despacho de Comunicaciones y Estrategia de Casa Presidencial.

El organigrama de dicho ente estatal es vertical, iniciando por el Despacho de Comunicaciones y Estrategia de Casa Presidencial, seguido de la Gerencia General de $\mathrm{TNH}$, producción general y finalmente dividiéndose en jefaturas (prensa, ingeniería, informática, transporte y bienes). Diariamente TNH transmite 16 horas de programación en directo y su parrilla de programación difunde géneros noticiosos, educativos y de entretenimiento.

Televisión Nacional de Honduras se define a sí misma como, "La televisión pública del pueblo hondureño con la misión de llegar a los hogares dentro y fuera de nuestras fronteras". (TNH, 2018) Tiene como misión fundamental el fortalecer la identidad nacional por medio de tres premisas: reflejar a Honduras en toda su diversidad, fortalecer la identidad nacional y conectar a los hondureños en todo momento y lugar. Esto induce a formularse las siguientes preguntas ¿Cumple TNH con las funciones de un canal público? ¿Promueve TNH el interés nacional? ¿Es TNH un canal de servicio público?

\subsection{Otros medios estatales}

Existen, asimismo otros tres canales que forman parte del sistema de canales públicos del Estado de Honduras (ver Tabla 1). El presidente del Congreso Nacional Mauricio Oliva en el 2017 presentó una iniciativa de ley para elevar a rango de canal estatal el canal de la Universidad Nacional Autónoma de Honduras UTV y manifestó (Díaz, 2017):

En ese sentido, el artículo uno del proyecto de decreto establece lo siguiente: Elévese a la categoría de canal estatal el canal de televisión de la Universidad Nacional Autónoma de Honduras, Canal Universitario de la Cultura; para que en el ámbito de la autonomía universitaria se integre al sistema de canales públicos del Estado de Honduras a fin de contribuir con el desarrollo económico y cultural del país. 


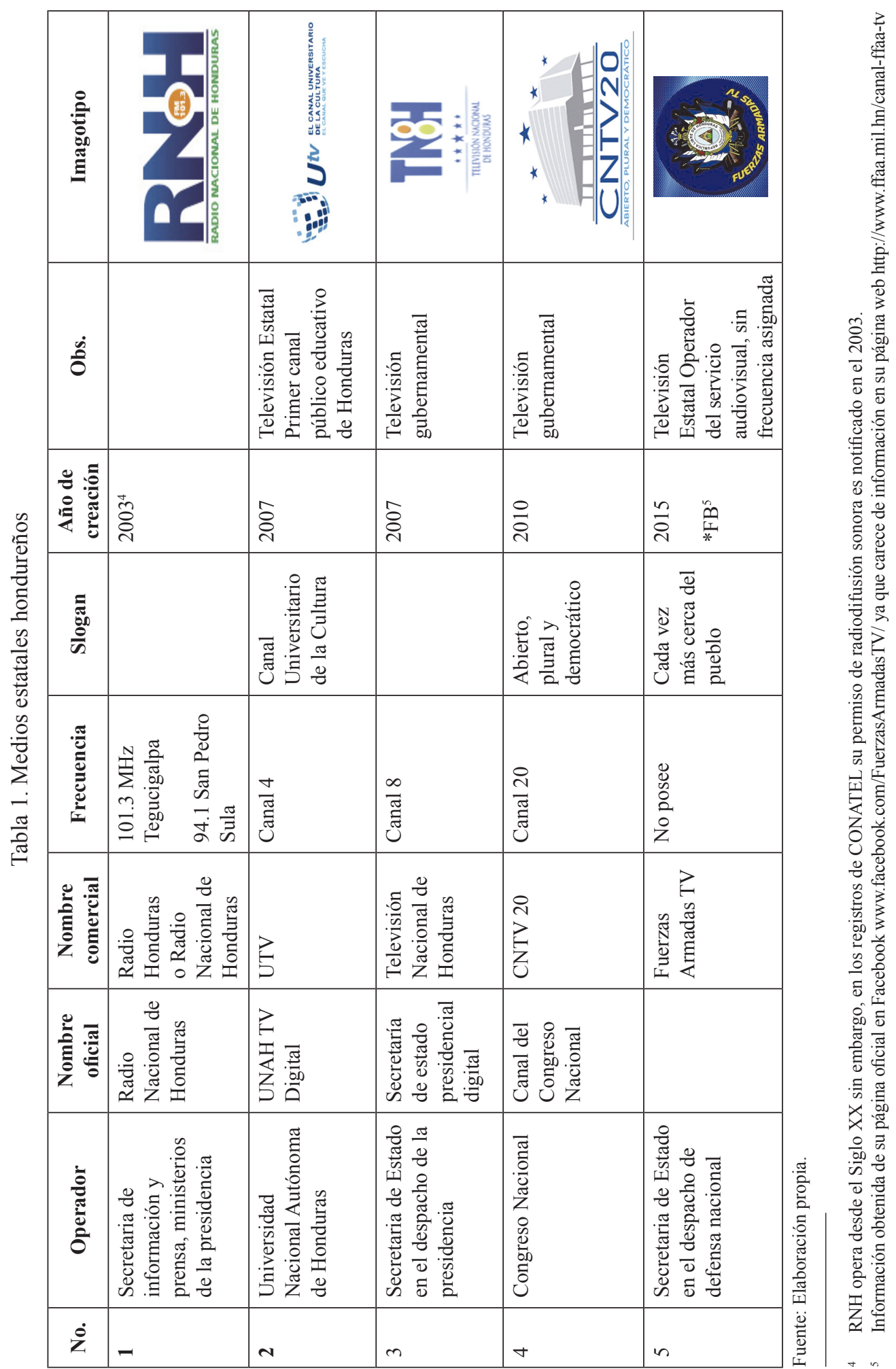


El término "canal público" es utilizado en reiteradas ocasiones por los entes gubernamentales y sus representantes; no obstante, como ya se analizó al inicio de este escrito, para ser un medio público se debe de cumplir con las premisas de servicio público e interés público, adicional a ello Pineda (2017) manifiesta:

Vale decir que no son administrados en base a una programación consensuada con las audiencias o sectores sociales, no parecen representar un espejo de la realidad hondureña, su función social e inclusiva hacia las minorías y etnias, medio ambiente, educación, salud, partidos políticos, sociedad civil, cultura, deportes u otros que todavía no se dejan ver y oír en estos medios. Queda mucho por avanzar en imparcialidad ideológica, objetividad, representación, inclusión. Es terreno dominado por la verdad oficial.

En segundo lugar, Fuerzas Armadas TV es administrada por las Fuerzas Armadas de Honduras (FFAA), transmite su señal en alta definición por medio de dos compañías de cable (Galavisión y Honduvisión), al ser un canal oficial está en el proceso de ser incorporado en todas las compañías de cable en el país como el canal 71. Se observa en su parrilla de programación programas noticiosos, religiosos, culturales y musicales, su contenido audiovisual promueve los valores cívicos y morales en la población centrándose en mostrar las actividades gubernamentales que se realizan. Las FFAA a su vez cuentan con una revista digital a la que se puede acceder libremente desde su página web bajo el lema "A la vanguardia informativa" donde difunde el quehacer de dicha institución.

Por último, CNTV 20, el canal del Congreso Nacional, también difunde sus contenidos en redes sociales (Youtube, Twitter y Facebook). De igual forma realizan publicaciones en digital por medio de boletines informativos -"Noticongreso"-, un semanario del Congreso Nacional que publica principalmente los pleitos entre diputados en el hemiciclo legislativo y el listado de los decretos aprobados en las sesiones. La programación de CNTV bajo el eslogan de "abierto, plural y democrático" se centra en difundir el trabajo que ejecuta el Congreso Nacional: emiten las sesiones de diputados, los programas del gobierno en turno y también emiten programas deportivos, noticieros y culturales.

La forma de financiación y estructura organizativa de estos medios de comunicación en Honduras es estatal o gubernamental, a excepción de UTV ya que al pertenecer a la universidad pública del país opera por medio de un consejo editorial universitario.

\section{Nuevo modelo de medios públicos en Honduras}

Los medios de comunicación tradicionales junto con el internet permiten hoy en día acceder a un mundo de información y conocimiento, es así como estamos llegando a construir nuevos paradigmas y nuevos modelos según las necesidades de nuestras sociedades debiendo tomar en consideración aspectos culturales, sociales y económicos de la región.

Un nuevo modelo de medios en Honduras debe de potenciar los medios de servicio público, con el protagonismo que su población y no el Gobierno en vigor les quiera otorgar. Un ejemplo de este proceso es la BBC de Gran Bretaña donde en sus inicios se narran las dificultades de lucha de poder político que enfrentaron, algunos 
ejemplos: huelga general de 1926, prohibición para discutir temas controvertidos en 1928, regla de los catorce días en 1956, etc. (Curran, 2005:16). Actualmente dicho ente superó parte de esas dificultades y en este momento goza de credibilidad sobresaliente con un nivel de satisfacción del $75 \%$, si bien reconocen que aún queda mucho trabajo por hacer seguirá siendo un modelo de medio público. Su publicación Green Paper detalla (Department for Culture, 2005:5):

The $\mathrm{BBC}$ needs to change to meet the public's concerns. While the $\mathrm{BBC}$ has a high overall satisfaction rating (75\%), the public is far from uncritical. $33 \%$ of people believe the $\mathrm{BBC}$ offers poor value for money. A third think its TV programmes are getting worse. People question the number of repeats, the amount of on-air trailing for BBC programmes, the perception of 'dumbing down' and the lack of accountability to licence fee payers.

Existen tres puntos prioritarios a destacar del éxito de la BBC. En primer lugar, su población ha apoyado, participado y defendido sus medios públicos, actualmente subvencionan este ente por medio del pago del canon ${ }^{6}$, reduciendo así la injerencia del gobierno y poseen el Green Paper, investigación que busca reflejar la opinión de la ciudadanía para proponer posteriormente reformas. En segundo, cuentan con una protección legal por medio de marcos regulatorios, el Royal Charter y framework agreement. Y como tercer punto cuentan con el OFCOM, la oficina de comunicaciones que promueve los intereses generales de la población, defiende la competencia en el área de la televisión, radio y telecomunicaciones en materia de regulación en el Reino Unido.

Siguiendo el referente de la BBC en este campo, para que exista un nuevo modelo de medios públicos en Honduras debe existir en primer lugar una Ley General de Comunicación, Información y Telecomunicaciones que promueva los valores de libertad bajo parámetros de desarrollo, respeto, identidad, equidad, acceso, pluralismo, cultura y valores. En la publicación "Hacía un nuevo modelo televisivo" se plantea (Domènec, 2009:20):

El diseño y la construcción del sistema del futuro residen, en gran medida, en las claves del modelo de nuestras democracias... Un nuevo modelo estará ligado a las sociedades de la información. Un nuevo modelo que muy pocos lo ponen en duda estará vinculado íntimamente a la que ya es materia prima principal del progreso sostenible: el conocimiento.

Es por ello por lo que a continuación se enlistan una serie de actividades que se podrían realizar con el fin de alcanzar un nuevo modelo de medios públicos hondureños:

- Realizar por medio de la academia foros, conversatorios y congresos sobre "el servicio público para todos", con el fin de crear una conciencia ciudadana

En Honduras el término Canon según la resolución legal NR016/01 de CONATEL se establece como un régimen especial de tarifas para organizaciones sin fines de lucro y se aplica para que el pago del derecho de permiso y canon radioeléctrico corresponda al monto equivalente al $10 \%$ de las respectivas tarifas vigentes al momento de realizar el pago. 
de la importancia que tienen los medios públicos para salvaguardar la democracia, libertad de expresión e información.

- Crear una Ley General de Comunicación, Información y Telecomunicaciones con la participación de la población y grupos prioritarios.

- Crear el Consejo Audiovisual hondureño con la participación de la academia, sociedad civil, Iglesia, un representante internacional y medios de comunicación que velen por el cumplimiento de la normativa bajo parámetros legales, éticos y morales.

- Rendir informes de gestión e investigación de mercados para promover la transparencia y el valor del servicio público.

- Realizar concursos públicos para elegir a los directivos y el personal que trabajará en los medios de comunicación públicos.

- Crear un directorio de medios públicos; conformado por representantes ejecutivos, académicos y de la sociedad civil que velen por la independencia gubernamental de la radio y televisión pública, descentralizando así su administración de Casa Presidencial.

- Asignar anualmente y de manera constante una partida presupuestaria estatal para los medios públicos con el fin que estos puedan realizar una planificación creativa de contenidos para ofrecer una programación diferenciada de las cadenas privadas, esto mediante decreto legislativo.

- Promover la participación ciudadana por medio de la Ley General de Comunicación, Información y Telecomunicaciones donde los ciudadanos puedan solicitar espacios en las cadenas públicas.

\section{Conclusiones}

Televisión Nacional de Honduras y Radio Nacional de Honduras se definen como medios públicos porque se financian con fondos nacionales por medio de la asignación de una partida presupuestaria de Casa Presidencial y por tener el amparo de alguna legislación, pero dado el análisis realizado en este artículo TNH y RNH se definirían como medios gubernamentales ya que su función principal desde su nacimiento ha sido el de promover fines políticos y propagandísticos, no se fundaron bajo la premisa del servicio público y han funcionado hasta la fecha como medios de comunicación oficial del Gobierno en vigor no teniendo ninguna autonomía o independencia.

Estos medios gubernamentales no realizan periódicamente estudios de mercado y mediciones de audiencias de su medio dadas las inversiones financieras que se requieren y por otra parte estos medios no aparecen dentro de las mediciones comerciales de rating del país. Hasta la fecha no se ha logrado obtener una mejor cercanía con la población, y no se ha cumplido a cabalidad con la función de servicio publico ya que no ofrecen dentro de su parrilla de programación contenidos variados y diversos que integren a todas las etnias y comunidades con una cobertura nacional. La dura realidad es que no son líderes en materia de telecomunicaciones en el país y muy poco se hace desde esta plataforma para fortalecer la identidad nacional hondureña.

El Gobierno tiene el deber de trabajar bajo el concepto del bien común, regulando y supervisando que las normativas y legislaciones se cumplan por medio de los entes correspondientes sin llegar a violentar el derecho de la mayoría o a coartar el derecho ineludible de la libertad de expresión. 
Claro está que los procesos de cambios y reformas no se realizan en cortos períodos de tiempo, sin embargo, es necesario iniciar con pequeñas acciones que llevarán a tener unos medios públicos que contribuyan a la democratización de la nación reforzando los valores sociales y culturales, donde se respete el acceso a la información y la pluralidad sea un baluarte.

Un nuevo modelo de medios públicos será posible solo con la participación de los diferentes sectores de la sociedad, quienes tengan consciencia que la comunicación y estar informado bajo diferentes puntos de vista es un derecho. Las trasformaciones en la sociedad son una constante, debido al cambio y avances tecnológicos a los que se está expuesto. Es necesario que surja un nuevo liderazgo para desarrollar estrategias donde los medios públicos puedan convertirse en referentes del sector.

Los ciudadanos hondureños son quienes deben de defender estos medios para que se conviertan en verdaderas plataformas de servicio público y posean una independencia genuina. Así mismo se considera necesario el impulso de movimientos sociales académicos. Es la sociedad civil y la academia quienes deben de exigir a los gobiernos el apoyo a estas plataformas de comunicación bajo nuevos modelos y paradigmas de los medios públicos con el propósito de crear una mayor conciencia en la población de los derechos y obligaciones que tienen como ciudadanos.

\section{Referencias bibliográficas}

Casa Presidencial. (2018). Radio Nacional de Honduras. Disponible en: http://www.rnh.gob. $\mathrm{hn} / \mathrm{index} \cdot \mathrm{html}$

CONATEL (2017). Disponible en: http://www.conatel.gob.hn/

Cuilenburg, J. van and D. M. (2003). Media Policy Paradigm Shifts. European Journal of Communication, vol. 18, $\mathrm{n}^{\circ}$ 2. https://doi.org/10.1177/0267323103018002002

Curran, J. (2005). Medios de comunicación y poder en una sociedad democrática. España: Barcelona, Hacer.

Department for Culture, M. and S. (2005). Review of the BBC's Royal Charter A strong BBC, independent of government. Great Britain. Disponible en: http://news.bbc.co.uk/2/shared/ bsp/hi/pdfs/02_03_05_bbcgreen.pdf

Despacho de comunicación y estrategia. (2018). Estrategias y comunicaciones. Disponible en: http://www.estrategiaycomunicaciones.gob.hn/mision

Díaz, E. (2017). Congreso Nacional eleva a rango estatal al canal de la UNAH, UTV. El Tiempo. Disponible en: https://tiempo.hn/congreso-nacional-eleva-rango-estatal-al-canal-la-unah-utv/

Domènec, M. (2009). Hacia un nuevo modelo televisivo (1st ed.). Barcelona (España): Gedisa, S.A.

Espectro, S. integrado de telecomunicaciones y administración del. (2017). SITAE. Disponible en: http://200.107.120.45/SIGERPAC/Radiodifusion/MapaRD/OperadoresRDTV. aspx

Group of specialists on public service media in the information society. (2007). Helsinki: Counsil of Europe.

Honduras, P. J. de. (1982). Constitución de la república de Honduras 1982 (DECRETO №). Tegucigalpa, Honduras: Diario Oficial La Gaceta. Disponible en: http://www.poderjudicial.gob.hn/CEDIJ/Leyes/Documents/ConstitucionRepublicaHonduras.pdf 
López Cepeda, A. M. (2015). Nuevos y viejos paradigmas de la Televisión Pública. (P. Crespo, Ed.) (1st ed.). Salamanca: Comunicación Social ediciones y publicaciones.

Mastrini, G. (2000). ¿Televisión estatal o gubernamental? Edición Cono Sur, 12, 32-33. Disponible en: https://www.insumisos.com/diplo/NODE/2325.HTM

Mastrini, G. (2011). Medios públicos y derecho a la comunicación. Revista Herramienta, 47. Disponible en: http://www.herramienta.com.ar/revista-herramienta-n-47/medios-publicos-y-derecho-la-comunicación

Mediadem. (2012). Media freedom and independence in 14 European countries: A comparative perspective. European Union: Mediadem.

Pasquiali, A. (1991a). El orden reina: Escritos sobre comunicaciones. (M. A. Editores, Ed.). Venezuela.

Pasquiali, A. (1991b). El orden reina: Escritos sobre comunicaciones (1st ed.). Caracas, Venezuela: Monte Avila Latinoamericana.

Pineda, M. (2009). Pensamientos Maupinianos. Disponible en: http://www.pensamientosmaupinianos.com/2009/09/el-futuro-de-la-tv-en-honduras.html

Pineda, M. (2017). Cuando los elefantes pelean. Disponible en: http://www.pensamientosmaupinianos.com/2017/12/cuando-los-elefantes-pelean.html

Prensa, L. (2008). Corte le quita al Gobierno la frecuencia de Canal 8. La Prensa. Disponible en: http://www.laprensa.hn/honduras/577932-97/corte-le-quita-al-gobierno-la-frecuencia-de-canal-8

Prensa, L. (2009). Rechazan anuncio de cuarta urna en Honduras. La Prensa. Disponible en: http://www.laprensa.hn/honduras/518052-97/rechazan-anuncio-de-cuarta-urna-en-honduras

Prensa, L. (2014). CSJ deja en firme la pertenecia de los canales 8 y 20 para el Estado. La Prensa. Disponible en: http://www.proceso.hn/component/k2/item/9198.html

RNH. (2018). Informe actualizado condición de Radio Honduras Año 2018. Honduras.

Semova, D. (2012). Entender la radiotelevisión pública. España, Madrid: Liberlibro.

TNH. (2018). ¿Quiénes somos? Disponible en: http://tnh.gob.hn/quienes-somos/

Tremblay, G. (1988). La noción del servicio público. Telos, 14, 57-63.

UNESCO. (2006). Radiotelevisión de servicio público: un manual de mejores prácticas. San José: UNESCO.

Vidal,A. (2006). El servicio público audiovisual y la construcción del Estado autonómico:RTVE 1980-2006. Universidad Autónoma de Barcelona. Disponible en: https://www.tdx.cat/bitstream/handle/10803/117784/av1de1.pdf?sequence=1\&isAllowed=y 